\title{
Major Sources Collaborate
}

\section{Y. Petroff, the Director General of the European Synchrotron Radiation Facility, summarises the fruitful collaboration between the world's three largest synchrotron sources.}

Third-generation hard X-ray machines such as the Advanced Proton Source (APS) in the USA, the European Synchrotron Radiation Facility (ESRF) and SPring- 8 in Japan have specific problems owing to their high energy ( 7,6 and $8 \mathrm{GeV}$, respectively). The very small size and small divergence of the sources, i.e., the very high brilliance of the beams, have forced the development of new techniques and new devices in the field of optics and detectors to ensure a high stability of the electron beam.

In May 1993, following an initiative by D. Moncton, the APS Director, the APS, ESRF and SPring-8 signed a framework agreement for collaboration in order to: - foster international scientific collabora-

\section{In-Vacuum Undulators}

The third-generation synchrotron radiation sources are mostly based on magnetic insertion devices called undulators. They produce narrow cones of intense radiation at wavelengths

$$
\lambda_{i}=\lambda_{\mathrm{w}}\left(1+K^{2} / 2\right) / 2 \gamma^{2} i
$$

where $i$ is the harmonics number, $\lambda_{w}$ the undulator period, $K=93.4 B_{0}[\mathrm{~T}] \lambda[\mathrm{m}]$ for a maximum magnetic field $B_{\mathrm{o}}$, and $\gamma=$ $E / m c^{2}$ where $E$ is the energy, $m$ the mass of

Fig. 1. A comparison of undulator spectra before (a) and after (b) spectrum shimming. Spectrum shimming increases the brightness at all harmonics and removes the background between peaks. The points correspond to an ideal field. tion among the three third-generation synchrotron radiation sources;

- combine each institution's expertise to accomplish unique scientific goals; - develop and use specialised knowledge and facilities on an international basis.

This is done through:

- Exchanges of technical information and of scientific personnel for short periods; - Research and development projects of common interest involving items such a Xray beam position monitors, detector development, X-ray optics and monochromators, enhanced cooling technology for front ends, small-gap in-vacuum undulator, continuous injection mode of storagering operation, and radio-frequency liners.

Fig. 2. A comparison of the source brilliance as specified by the ESRF Foundation Phase Report design goal and by the medium-term objectives (already achieved). It shows gains ranging from 10 to 100 in terms of brilliance from dipoles, wigglers and undulators. At high energy, the spectrum shimming of undulators has increased dramatically their exploitable range.

the electron and $c$ the speed of light. The useful number of harmonics (peaks of intensity) depends strongly on the low emittance (the product of the electron beam size and the electron divergence) which can be achieved and on the quality of the undulators. The technique of shimming the undulators to tailor the spectrum [J. Chavanne \& P. Elleaume, Synchrotron Radiation News 8 (1995) 18] which was introduced recently at the ESRF allows us to work with harmonics as high as the 15 th. This is illustrated in Fig. 1 showing undulator spectra before and after shimming.

Another very important parameter is the size of the undulator gap because the magnetic field is produced with NdFeBbased permanent magnets. With a $20 \mathrm{~mm}$ gap and horizontal and vertical emittances of $7 \times 10^{-9} \mathrm{mrad}$ and $7 \times 10^{-10} \mathrm{mrad}$, the maximum useful X-ray energy was $25 \mathrm{KeV}$. With the emittances of $4 \times 10^{-9} \mathrm{mrad}$ and $4 \times 10^{-11}$ mrad achieved today at the ESRF and a 10 $\mathrm{mm}$ gap, the maximum energy has been raised to $70 \mathrm{KeV}$ (Fig. 2). However, to go below a $10 \mathrm{~mm}$ gap and above $70 \mathrm{KeV}$ one has to place the permanent magnets in a vacuum because it is very difficult to pump
Third-Generation Synchrotron Sources

European Synchrotron Radiation Facility $(6 \mathrm{GeV})$

- Official user service started on 1 September 1994

- 30 public beamlines by end of 1998

- 9 group beamlines operating, planned,under design

Advanced Proton Source (Argonne, USA; $7 \mathrm{GeV}$ )

- 1st electron beam in March 1995

- Now being commissioned

- 1st experiment in early 1996

SPring-8 (Harima, Japan; $8 \mathrm{GeV}$ )

- Under construction

- 1st electron beam in 1997

- User operation in 1998

- An annual technical workshop on accelerator and beamline topics organized by one of the facilities.

As the ESRF is the first facility in operation, results obtained in Grenoble in various fields are being usefully applied within the framework of the collaboration at APS and SPring-8. To date, the collaboration has been quite useful (see below) and once the three sources are in operation, it could be envisaged to go beyond the present scope of collaboration and exchange beam time in an area not covered by a centre.

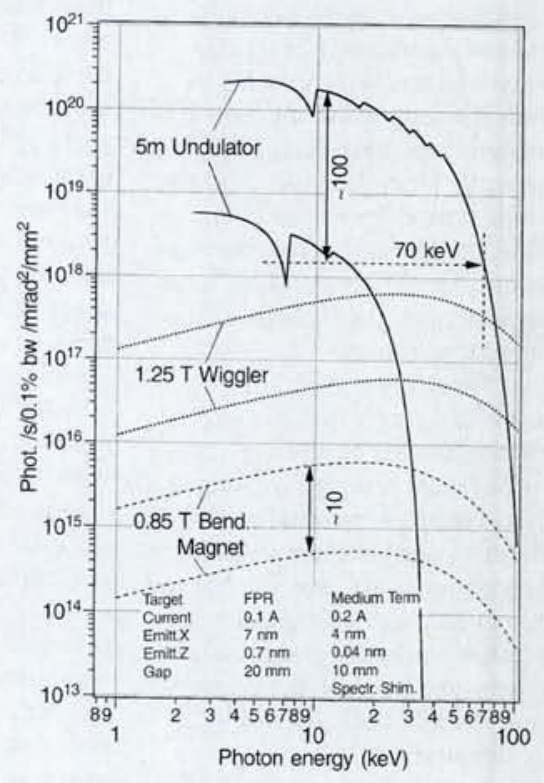

a 5-m long vacuum chamber with a 5-mm gap. In-vacuum undulators exist already at the KEK high-energy physics laboratory in Japan [S. Yamamoto et al., J. Appl. Phys. 74 (1993) 502] and they give very good results. However, owing to the much larger emittance, it has not been possible to study the possible demagnetisation of the magnets which has been observed at the ESR [J. Chavanne \& P. Elleaume]. In a collaboration project, SPring- 8 is building an invacuum undulator that will be installed and tested at the ESRF this summer. 


\section{Radio-Frequency Liners}

Bellows are needed between various parts of an accelerator. However, owing to changes in the cross-section, they act as an RF cavity thereby inducing beam oscillations. The RF liners which are placed around the beam in order to ensure pumping are thus subjected to heating effects within bellow sections. Difficulties have been encountered in bellow sections in the few bunch/high current mode on existing storage rings and are anticipated for new machines. At the ESRF, the stored current in the 16-bunch mode is limited by significant heating effects, and the subsequent bursting under pressure of liners in bellow sections. Several designs which may solve the problem have been developed at the APS and the ESRF and are now under test.
X-ray Beam Position Monitors

At the undulator beamlines, it is very important to measure precisely the position of the X-ray beam using beam-position monitors. Optical detectors placed at the end of a beamline work well for bending magnet and wiggler sources, and for undulators with a fixed gap. However, they have been found to be very sensitive to spurious radiation from bending magnets, both up- and down-stream of the undulator. They give a reading of the centre-ofmass position of the photon beam which depends on the undulator gap. Consequently, conventional optical detectors cannot be used for variable-gap undulators. An ESRF-APS collaboration is therefore designing and testing a new generation of X-ray monitors.

\section{Monochromators}

The APS will operate at $7 \mathrm{GeV}$ instead of the ESRF's $6 \mathrm{GeV}$ and as the power increases quadratically with the energy, it is necessary to perform high-power tests of some of the APS's optics devices. A cooled Bragg monochromator has been successfully tested at the ESRF up to $186 \mathrm{~W}$ and at an average power density of $520 \mathrm{~W} / \mathrm{mm}^{2}$. Diamond deposited as a 200-300 $\mu \mathrm{m}$ layer by vapour deposition is an almost ideal material for monochromators on undulator beamlines, owing to its low X-ray absorption, low thermal expansion and high heat conductivity. Water-cooled diamond monochromators can cope with 280 $\mathrm{W}$ of total power $\left(3.4 \mathrm{KW} / \mathrm{mm}^{2}\right)$. Four are under construction at the ESRF, one being for the APS.

\section{Roundup}

\section{Energy Amplifier}

A team led by C. Rubbia will start up in April a CERN experiment - cofunded by the European Union's Framework Programme and international sources - to further explore the feasibility of the "energy amplifier" concept in which heat is produced when neutrons originating from spallation by a proton accelerator beam are multiplied by fission in a subcritical assembly. Lead is an excellent material for the spallation target since neutrons have a remarkably large migration length with only a few weakly capturing resonances. Fissile material would be located between the spallation source and the outer boundary of the lead target. The experiment aims to study neutronics in a large lead block in order to verify simulations which are sensitive to subtle material properties such as the resonant captures of lead. Meanwhile, the collaboration is seeking international support for an accelerator that would be the first stage of a prototype system. The Trieste government is reportedly close to approving an approximately 20 MECU contribution for an accelerator that would be built at the ELETTRA synchrotron.

\section{Austron Green Light}

Following completion last year of a feasibility study financed by the Austrian Ministry for Science and Research, the Austrian Government has given the green light for the proposers of the 4200 MATS Austron neutron spallation source to discuss with other countries possible participation. Austria would contribute to the capital cost as well as approximately one-third of the 400 MATS annual operating budget. The government has requested that the European Commission, OECD and the European Science Foundation be involved. The proposers believe that the facility would meet the increasing need for neutron beam time in offering services within a multidisciplinary environment to small groups from industry and universities. A light-ion source will emphasise cancer therapy.

\section{Space Spectrometer}

Italy and Switzerland have approved contributions totalling some 7 MSUS to an international collaboration that will build and operate the approximately 20 M\$US Alpha Magnetic Spectrometer (AMS) detector for cosmic particles and nuclei - the first large experiment equipped with a magnet to be placed in orbit. This follows last year's announcement that the US Department of Energy would contribute some 2 M\$US. Preliminary, but valuable, data will be generated when most of the detector, which is based on a 2 $\mathrm{t}, 1 \mathrm{~m}$ in diameter, $0.8 \mathrm{~m}$ long cylindrical magnet, is tested under space conditions for 10 days aboard a Space Shuttle in May 1998. The complete detector will be sited aboard the space station Alpha for 3 years starting in 2001.

\section{ESRF Development}

The Council of Grenoble's European Synchrotron Radiation Facility has asked the facility's management to submit by June 1996 a multi-scenario plan for medium- and long-term development based on continuous rather that step-wise development.

\section{Megascience Groups}

Following the ministerial decision last autumn to give the OEDC Megascience Forum more powers, the Forum has set up working groups to plan global cooperation in neutron sources, compatibility between biological databases, and measures to improve collaboration.

\section{CNRS Protest}

Some two-thirds of the 150 directors of France's CNRS laboratories have protested budget restrictions whereby unspent 1995 funds have been held back following the emptying of laboratory accounts for auditing. The problem is that authorised budgets were not covered by credits so the CNRS has acquired a large deficit.

\section{Department of Physics}

Technical University of Denmark (DTU) in Lyngby (Copenhagen) invites applications for positions as

\section{Associate Professor in Structural Physics}

covering the relationship between structure, structure formation, and function in structurally determined physical phenomena, In particular the professor should take part in the build-up of activities in molecular biophysics. It will be advantageous if the applicant can contribute to or support the use of available TEM and AFM facilities. The applicant's possibility of creating synergy between molecular biophysics and existing research on metal clusters and pattern formation in general will be considered.

In addition to research activities the professor will participate in teaching elementary and advanced physics to engineering students and in supervision of Masters and Ph.D. projects. For information including the full text of the announcement contact the group leader Jakob Bohr. E-mail: jakob.bohr@fysik.dtu.dk. It is the policy of DTU to encourage both male and female candidates to apply. Persons wishing to inquire about other possibilities for working on related projects may contact Prof. Bohr.

Applications should be submitted to Rektor Hans Peter Jensen, DTU, DK-2800 Lyngby, Denmark, no later than May 31, 1996, at noon sharp. 